\title{
Effect of substituting tapioca starch with various high protein legume flours on the physicochemical and sensory properties of keropok lekor (Malaysian fish sausage)
}

\author{
${ }^{1}$ Nur Liyana, N., ${ }^{2}$ Nor-Khaizura, M.A.R. and ${ }^{1 *}$ Ismail-Fitry, M.R. \\ ${ }^{1}$ Department of Food Technology, Faculty of Food Science and Technology, Universiti Putra Malaysia, \\ 43400, Serdang, Selangor, Malaysia \\ ${ }^{2}$ Department of Food Science, Faculty of Food Science and Technology, Universiti Putra Malaysia, 43400, \\ Serdang, Selangor, Malaysia
}

\begin{abstract}
Article history:
Received: 29 July 2018

Received in revised form: 14

September 2018

Accepted: 18 September 2018

Available Online: 28

September 2018

\section{Keywords:}

Keropok lekor,

Malaysian fish sausage,

Chickpea flour,

Soybean flour,

Mung bean flour,

Kidney bean flour
\end{abstract}

DOI:

https://doi.org/10.26656/fr.2017.3(1).217

\begin{abstract}
Keropok Lekor (Malaysian fish sausage) produced in Kuala Lumpur (KLKL) due to its lower fish content is perceived to have lesser nutritional values especially protein against Keropok Lekor from Terengganu (KLT). This study formulated new Keropok Lekor by substituting tapioca starch with high protein flour, which was chickpea (KLCP), soybean (KLSB), mung bean (KLMB) or kidney bean (KLKB) and evaluated against the KLKL (negative control) and KLT (positive control). All the new formulated Keropok Lekors had higher protein contents $(\mathrm{p}<0.05)$ compared to KLKL and not significantly different compared to KLT $(\mathrm{p}>0.05)$. The carbohydrate, moisture, fat and ash results were acceptable compared to both controls. Majority of the new formulated Keropok Lekors resulted with higher cooking yield compared to KLT $(\mathrm{p}<0.05)$ but not significant against the KLKL $(\mathrm{p}>0.05)$. From the sensory evaluation, the colour preference was similar for all Keropok Lekor formulations matching the CIEL*a*b colour values. There was no difference $(p>0.05)$ in aroma preference between all Keropok Lekor formulations. KLKL and KLMB were the highest in chewiness of sensory attribute, relatively to higher chewiness value from Texture Profile Analysis. Juiciness preference favoured KLT, KLKL and KLMB compared to other formulations. Overall, panellists preferred KLCP and KLMB from the new formulations since they are almost similar to KLT and KLKL $(p<0.05)$. This study indicates that legume flours could substitute tapioca starch in Keropok Lekor to improve the nutritional values but retain the quality attributes.
\end{abstract}

\section{Introduction}

Keropok Lekor is a very popular fish sausage-like product eaten by all races and communities in Malaysia. The name Keropok Lekor originates from Terengganu and also known as Keropok Batang in Kelantan and Keropok Tongkol in Pahang (Jamilah, 1983). Among the types of fish used in Keropok Lekor processing are wolf herring, sardine, threadfin bream, yellow goatfish, purple -spotted bigeye, mackerel and many more (Jamilah, 1983; Malaysian Fisheries Department, 2014; Hatta, 2015). The basic ingredients of Keropok Lekor are minced fish flesh, sago flour, tapioca starch, sugar, salt, crushed ice and permitted flavour enhancer (Malaysian Fisheries Department, 2014). The flour and/or starch are used as the fish flesh filler in the Keropok Lekor formulation.

The Keropok Lekor industry in Malaysia involves processing and production from the backyard to modern small or medium-scale production (Tang et al., 2014). The demographics of Keropok Lekor production in Malaysia can be focused on east coast states such as Terengganu, Kelantan and Pahang, where the fish source is abundance (Ahmad et al., 2011). Terengganu has been the main producer of Keropok Lekor, which originally invented by fishermen and has become their major economic income (Hatta, 2015). Keropok Lekor from east coast states is the most popular in Malaysia for its taste and texture due to the high amount of fish and less amount of flour used. Keropok Lekor also has been produced in other states in Malaysia such as in the central region (Kuala Lumpur and Selangor) but with a lower amount of fish and high amount of flour. As a result, the nutritional values especially protein of Keropok Lekor from Terengganu are considered better compared to Keropok Lekor from Kuala Lumpur. Fresh Keropok Lekor has a short shelf life of one day when 
exposed to room temperature (Embong et al., 1990). Therefore to transport fresh Keropok Lekor from Terengganu to Kuala Lumpur with a distance of about $450 \mathrm{~km}$ is very inefficient.

Chickpea, soybean, mung bean and kidney bean have a high amount of protein and other nutritional benefits. Some of these legumes were used as flour and incorporated into meat-based products such as mung bean flour into buffalo meat product as meat extenders (Kenawi et al., 2009), chickpea flour incorporated in sausages (Verma et al., 1984), red kidney beans as protein isolate based product for food applications (Hayat et al., 2014) and the development of soybean meatballs (Odiase et al., 2013). However, there is still a lack of research carried out on Keropok Lekor produced with these legume flours. The incorporation of these legumes flour could reform the formulation of Keropok Lekor currently being produced in Kuala Lumpur to improve the nutritional values but retains other quality aspects. Therefore, the aims of this study were to substitute the tapioca starch with four selected highprotein legume flours (chickpeas, soybeans, mung beans and kidney beans) into Keropok Lekor and to study the effect on their physicochemical properties and sensory acceptance against the positive control (Keropok Lekor from Terengganu) and negative control (Keropok Lekor from Kuala Lumpur).

\section{Materials and methods}

\subsection{Materials}

Longtail tuna (Thunnus Tongol) and Indian mackerel (Rastrelliger kanagurta) were purchased from Pasar Borong Selangor, Sri Kembangan, Selangor. Legumes (chickpeas, soybeans, mung beans and kidney beans) were purchased from E Link Mart Cash and Carry, Sri Kembangan, Selangor. Cooking oil, tapioca starch, sago flour, salt, sugar, and monosodium glutamate (MSG) were purchased from Giant Hypermarket, Sri Kembangan, Selangor.

\subsubsection{Deboning of fish}

Both Longtail tuna (50\% of total fish) and Indian mackerel $(50 \%$ of total fish) were deboned by using a deboner machine (World Foodtech Sdn Bhd, Klang, Selangor, Malaysia). The Longtail tuna and Indian mackerel were gutted, and visceral mass was removed. Both fish were headed and rinsed under running water. Fish were subjected to a deboner machine, and minced fish were obtained. Minced fish were packed in an airtight container and stored in a freezer $\left(-18^{\circ} \mathrm{C}\right)$ until further processing.

\subsubsection{Legumes flour processing}

Legumes were processed into flours according to a method performed by Blessing and Gregory (2010). Undehulled legumes (chickpeas, soybeans, mung beans and kidney beans) were rinsed under running water. Legumes were placed on trays and dried in a Food Cabinet dryer at $60^{\circ} \mathrm{C}$ for $2 \mathrm{hrs}$. After dried, legumes were left to cool down at $25^{\circ} \mathrm{C}$. Ultra-Centrifugal mill (Retsch, ZM 200, Haan, Dusseldorf, Germany) were used to grind legumes into fine raw flours. Legume flours were stored in an airtight container until further processing.

\subsubsection{Keropok Lekor processing}

Keropok Lekor processing was carried out according

Table 1. Formulations for Keropok Lekor produced with different types of flour.

\begin{tabular}{|c|c|c|c|c|c|c|}
\hline Ingredients & $\begin{array}{l}\text { Keropok Lekor } \\
\text { Terengganu } \\
(\text { KLT })\end{array}$ & $\begin{array}{c}\text { Keropok } \\
\text { Lekor Kuala } \\
\text { Lumpur } \\
\text { (KLKL) }\end{array}$ & $\begin{array}{l}\text { Keropok } \\
\text { Lekor } \\
\text { Chickpea } \\
(\text { KLCP) }\end{array}$ & $\begin{array}{l}\text { Keropok Lekor } \\
\text { Soybean } \\
\text { (KLSB) }\end{array}$ & $\begin{array}{l}\text { Keropok Lekor } \\
\text { Kidney bean } \\
(\text { KLKB })\end{array}$ & $\begin{array}{c}\text { Keropok } \\
\text { Lekor Mung } \\
\text { bean } \\
\text { (KLMB) }\end{array}$ \\
\hline & $\%$ & $\%$ & $\%$ & $\%$ & $\%$ & $\%$ \\
\hline $\begin{array}{l}\text { Fish Flesh }(50 \% \\
\text { Indian Mackerel and } \\
50 \% \text { Longtail tuna })\end{array}$ & 70 & 50.18 & 50.18 & 50.18 & 50.18 & 50.18 \\
\hline Sago flour & 6.94 & 17.56 & 17.56 & 17.56 & 17.56 & 17.56 \\
\hline Tapioca Starch & 6.94 & 17.56 & - & - & - & - \\
\hline Chickpea Flour & - & - & 17.56 & - & - & - \\
\hline Soybean Flour & - & - & - & 17.56 & - & - \\
\hline Kidneybean Flour & - & - & - & - & 17.56 & - \\
\hline Mung bean Flour & - & - & - & - & - & 17.56 \\
\hline Salt & 1.27 & 1.27 & 1.27 & 1.27 & 1.27 & 1.27 \\
\hline Sugar & 0.76 & 0.76 & 0.76 & 0.76 & 0.76 & 0.76 \\
\hline MSG & 0.15 & 0.15 & 0.15 & 0.15 & 0.15 & 0.15 \\
\hline Ice & 13.94 & 13.94 & 13.94 & 13.94 & 13.94 & 13.94 \\
\hline Total & 100 & 100 & 100 & 100 & 100 & 100 \\
\hline
\end{tabular}


to Murad et al. (2017) with slight modifications. The formulation for Keropok Lekor processing was performed according to the Malaysian Fisheries Department (2014) with slight modifications. All ingredients were weighed according to the formulation provided in Table 1 . The ingredients were mixed in a mixer machine (K3 Mini-Luxury Cutting Machine, Kinn Shang Hoo Iron Works, Taiwan) for 7 mins until homogenized. The dough was shaped and rolled into 10 $\mathrm{cm}$ long sausage-like. Keropok Lekor dough then was boiled at $100^{\circ} \mathrm{C}$ for 25 mins. The boiled Keropok Lekors were placed on trays and left to cool down to the room temperature. Each Keropok Lekor was packed in an airtight container and kept in a freezer at $-18^{\circ} \mathrm{C}$ before further analyses. The frozen Keropok Lekor samples were thawed for $1 \mathrm{hr}$ and used for proximate analysis. Meanwhile, the thawed Keropok Lekor samples were further fried at $180^{\circ} \mathrm{C}$ in vegetable oil for 3 mins, left to cool down at room temperature for 5 mins and used for physical analysis and sensory evaluation. KLT and KLKL were used as a positive and negative control, respectively.

\subsection{Proximate analysis}

Proximate analysis to determine moisture, protein, fat, carbohydrate and ash content of all Keropok Lekor formulations was carried out based on the standard methods of the Association of Official Analytical Chemists, AOAC (2002). Moisture contents were analysed by using oven drying method, protein contents were analysed by using the Micro Kjeldahl method, fat contents were analysed by using Soxhlet method, ash contents were analysed by using the dry method and carbohydrate contents were obtained through calculations.

\subsection{Cooking yield}

Cooking yield was measured as the percentage of cooked Keropok Lekor weight compared to the original weight before cooking (Santana et al., 2013). The analyses were carried out in triplicates for each of Keropok Lekor formulations based on the formulation below.

Cooking yield $=$ (Weight of cooked Keropok Lekor/ Weight of raw Keropok Lekor) $/ 100 \%$

\subsection{Colour analysis}

The measurement of colour for all six Keropok Lekor formulations were carried out according to the method performed by Rahman et al. (2007) by using HunterLab Ultrascan Spectrocolorimeter (Hunter Associate Laboratory Inc., Reston, USA) with CIEL*a*b* colour system $(\mathrm{L}=$ lightness (black $\mathrm{L}=0$; white $\mathrm{L}=100),+\mathrm{a}=$ redness; $-\mathrm{a}=$ greenness, $+\mathrm{b}=$ yellowness, and $-b=$ blueness). All six formulations were thawed for $1 \mathrm{hrs}$, sliced into $2.5 \mathrm{~cm}$ thickness and fried with vegetable oil for 3 mins and left to cool down at room temperature for 5 mins. The measurements were run in triplicates for each Keropok Lekor formulations.

\subsection{Texture profile analysis}

Texture Profile analysis (TPA) conducted for all six Keropok Lekor formulations were measured using Texture Analyser (TA-XT2, Stable Micro Systems, Ltd, Surrey, UK) following the method conducted by Hayes et al. (2005). Parameter settings were; load cell: $25 \mathrm{~kg}$, compression platen: P.75, constant rate: $1 \mathrm{~mm} / \mathrm{s}$, trigger force: $10 \mathrm{~g}(2 \mathrm{~s})$, pre-test and post-test speed: $3 \mathrm{~mm} / \mathrm{s}$ and return distance: $35 \mathrm{~mm}$. All six Sliced of Keropok Lekor samples $(2.5 \mathrm{~cm})$ was placed horizontally on the platform and compressed. Samples were subjected to hardness, springiness, cohesiveness, and chewiness. The measurements were run in triplicates for each Keropok Lekor formulations.

\subsection{Sensory evaluation}

Sensory evaluation was carried out by using the acceptance test performed by Prabpree and Pongsawatmanit (2011) with 30 untrained panellists. The hedonic scale used was 7 (extremely like) until 1 (extremely dislike). Panellists were served with $1.5 \mathrm{~cm}$ thickness of Keropok Lekor along with three-digit coded numbers in random order. All six Keropok Lekor formulations were evaluated based on the attributes of colour, aroma, chewiness, juiciness, aftertaste and overall acceptability.

\subsection{Data analysis}

The analysis of data was performed by using Minitab Software (Minitab 17.0 for Windows, Minitab, USA). All analyses were carried out in triplicates, and one-way analysis of variance (ANOVA) was used for the analytical variation. Data were presented as a mean \pm standard deviation. Tukeys' test was used for comparison of means with a level significance of 0.05 .

\section{Results and discussion}

\subsection{Proximate analysis}

The proximate analysis for all the Keropok Lekors is shown in Table 2. The highest protein content was obtained from Keropok Lekor soybean (KLSB) at $17.40 \%$. This follows by Keropok Lekor Terengganu (KLT), Keropok Lekor chickpea (KLCP), Keropok Lekor kidney bean (KLKB), Keropok Lekor mung bean (KLMB) and Keropok Lekor Kuala Lumpur (KLKL) at 
Table 2. Proximate analysis of Keropok Lekor produced with different types of flour.

\begin{tabular}{ccccccc}
\hline $\begin{array}{c}\text { Proximate } \\
\text { analysis }(\%)\end{array}$ & $\begin{array}{c}\text { Keropok Lekor } \\
\text { Terengganu } \\
(\text { KLT })\end{array}$ & $\begin{array}{c}\text { Keropok lekor } \\
\text { Kuala Lumpur } \\
(\text { KLKL) }\end{array}$ & $\begin{array}{c}\text { Keropok Lekor } \\
\text { Chickpea } \\
(\text { KLCP) }\end{array}$ & $\begin{array}{c}\text { Keropok Lekor } \\
\text { Soybean } \\
\text { (KLSB) }\end{array}$ & $\begin{array}{c}\text { Keropok Lekor } \\
\text { Mung bean } \\
(\text { KLMB) }\end{array}$ & $\begin{array}{c}\text { Keropok Lekor } \\
\text { Kidney bean } \\
(\text { KLKB) }\end{array}$ \\
\hline Protein & $17.01 \pm 0.44^{\mathrm{a}}$ & $10.11 \pm 1.10^{\mathrm{c}}$ & $16.14 \pm 0.44^{\mathrm{a}}$ & $17.40 \pm 1.18^{\mathrm{a}}$ & $13.22 \pm 1.10^{\mathrm{b}}$ & $15.36 \pm 0.93^{\mathrm{ab}}$ \\
Carbohydrate & $15.02 \pm 3.88^{\mathrm{c}}$ & $26.91 \pm 0.36^{\mathrm{a}}$ & $18.46 \pm 1.89^{\mathrm{bc}}$ & $19.12 \pm 1.97^{\mathrm{bc}}$ & $23.34 \pm 0.79^{\mathrm{ab}}$ & $20.58 \pm 2.26^{\mathrm{bc}}$ \\
Fat & $3.98 \pm 0.34^{\mathrm{c}}$ & $3.63 \pm 0.49^{\mathrm{c}}$ & $6.68 \pm 0.47^{\mathrm{a}}$ & $4.92 \pm 0.80^{\mathrm{bc}}$ & $5.44 \pm 0.30^{\mathrm{ab}}$ & $5.65 \pm 0.48^{\mathrm{ab}}$ \\
Moisture & $62.42 \pm 3.70^{\mathrm{a}}$ & $57.79 \pm 0.65^{\mathrm{ab}}$ & $56.50 \pm 2.06^{\mathrm{b}}$ & $56.44 \pm 0.62^{\mathrm{b}}$ & $55.99 \pm 0.43^{\mathrm{b}}$ & $56.63 \pm 1.31^{\mathrm{b}}$ \\
Ash & $1.56 \pm 0.19^{\mathrm{b}}$ & $1.56 \pm 0.19^{\mathrm{b}}$ & $2.22 \pm 0.19^{\mathrm{a}}$ & $2.11 \pm 0.19^{\mathrm{ab}}$ & $2.00 \pm 0.33^{\mathrm{ab}}$ & $1.78 \pm 0.19^{\mathrm{ab}}$ \\
\hline
\end{tabular}

${ }^{\mathrm{a}-\mathrm{c}}$ Mean values with different letters in the same row are significantly different $(P<0.05)$.

$17.02 \%, \quad 16.14 \%, \quad 15.36 \%, \quad 13.22 \%$ and $10.11 \%$, respectively (Table 2). According to the Malaysian Food Regulations 1985, fish keropok should not contain less than $15 \%$ protein content. All the new formulated Keropok Lekors had higher protein contents $(\mathrm{p}<0.05)$ compared to KLKL (negative control). This study proved that by substituting the tapioca starch with legume flour while retaining the same amount of fish flesh (50.18\%) used in the formulation, could increase the protein content in Keropok Lekors. Meanwhile, the protein contents in the new formulated Keropok Lekors were not significantly different compared to KLT (positive control) ( $p>0.05)$ except for KLMB. Therefore, the result shows that the substitution of tapioca starch with legume flour, retained the protein content even when the amount of fish flesh used for KLT is higher (70\%) compared to the new formulated Keropok Lekors (50.18\%). Keropok Lekor soybean (KLSB) had the highest protein content compared to other Keropok Lekor formulations. This can be related to the protein contents in the legumes themselves. Soybean flour has a protein content of $38.43 \%$ (Perera et al., 2013), chickpea flour has $24.61 \%$ (Wani and Kumar, 2007), mung bean flour ranging between 20.8-23.7\% (Abbas and Shau, 2007), and kidney bean seed has $25.78 \%$ of protein content (Perera et al., 2013). Analysis of five brands of commercial Malaysian fish sausages resulted in protein contents between $8.18-10.77 \%$ (Huda et al., 2012). The commercial Malaysian sausages were mainly composed of fish, minced fish, surimi, mixed salmon or tuna in its ingredients. Another study conducted by Murad et al. (2017), discovered that the protein content in the Keropok Lekors was between $8.92-10.80 \%$. This study proves that by substituting tapioca starch with legume flours in Keropok Lekor did effectively increase the protein content.

The carbohydrate content for all the new formulated Keropok Lekors was not significantly different $(p>0.05)$ compared to the KLT and significantly lower $(p<0.05)$ compared to the KLKL except for KLMB. The KLKL has the highest carbohydrate content $(26.91 \%)$ followed by KLMB (23.34\%). Previous literature have recorded that tapioca starch contains high carbohydrate content at 93.12\% (Seechamnanturakit and Karrila, 2015).
Meanwhile, carbohydrate content for Keropok Lekors formulated with legume flours could be influenced by chickpea flour at $64.60 \%$ (Alajaji and El-Adawy, 2006), soybean flour at $32.35 \%$ (Perera et al., 2013), mung bean flour at 55.74\% (Blessing and Gregory, 2010) and kidney bean flour at 56.7\% (Chaudhary and Sharma, 2013) of carbohydrate contents. Therefore, the substitution of legume flours did not affect the changes of carbohydrate content in Keropok Lekors due to the high carbohydrate content of tapioca starch compared to other legume flours. Nevertheless, fish sausage formulated with surimi powder with addition of hydrocolloids recorded carbohydrate content ranging between 13.15-13.46\% (Santana et al., 2013) which are lower compared to Keropok Lekors shown in this study. Carbohydrate content in all the new formulated Keropok Lekors is related to the presence of starch in the legume flours similar to what Huda et al. (2012) found, where the addition of starch and sugar results in the presence of carbohydrates in fish sausages.

The moisture content for all the new formulated Keropok Lekors was not significantly different compared to KLKL ( $p>0.05$ ) but significantly lower compared to KLT $(p<0.05)$. This result could be explained by the fish contents for each of the formulations. The KLT comprised of $70 \%$ minced fish, $6.94 \%$ sago flour and $6.94 \%$ tapioca starch. Meanwhile, other Keropok Lekor formulations comprised of $50.18 \%$ minced fish, $17.56 \%$ sago flour and $17.56 \%$ tapioca starch or other legume flours. The extra $28.3 \%$ of fish content in the KLT formulation could influence the higher moisture content compared to other formulations. This can be proven with the moisture content for Indian Mackerel and Longtail tuna detected is $70.95 \%$ and $76.8 \%$, respectively (Lakshmisha et al., 2008; Hajeb et al., 2009). While, lower moisture content was detected for tapioca, kidney bean, chickpea, soybean and mung bean at 5.89\% (Seechamnanturakit and Karrila, 2015), 8.12\% (Audu and Aremu, 2011), 8.40\% (Wani and Kumar, 2014), $5.20 \%$ (Perera et al., 2013), 12.07\% (Paul et al., 2011) respectively. The findings proved that the substitution of legume flours did not bring changes in the moisture content of Keropok Lekors and was due to the low moisture content in the legume flour itself. 
The fat content for all the new formulated Keropok Lekors was significantly higher $(\mathrm{p}<0.05)$ compared to the KLT and KLKL. Soybean, kidney bean, chickpea and mung bean has $21 \%$ (Perera et al., 2013), 1.5\% (Chaudhary and Sharma, 2013), 5.62\% (Arab et al., 2010 ) and $1.89 \%$ (Blessing and Gregory, 2010) fat content, respectively. The fat from the legumes may contribute to the increase of the fat content to the Keropok Lekor incorporated with the legume flour. Therefore, this study shows that the substitution of legume flours into Keropok Lekor did significantly increase the fat content due to the presence of high fat content in the legume flours itself. Meanwhile, the fat content of Keropok Lekors formulated with tapioca starch is mainly from the fish flesh. Nevertheless, these fats from legumes are unsaturated fat, which is the healthy fat. For example, the polyunsaturated fatty acids (linoleic and linolenic acids) from legumes such as soybeans can be crucial to human health (Hegstad, 2008).

KLCP increased significantly $(\mathrm{p}<0.05)$ in ash content at $2.22 \%$ compared to KLT and KLKL both at $1.56 \%$. However, KLSB, KLMB and KLKB did not differ significantly from KLT and KLKL $(p>0.05)$ at $2.11 \%, 2.00 \%, 1.78 \%$ respectively. Similar ash content results were shown by five Malaysian commercial brand fish sausages (Huda et al., 2012) ranging between 1.71$2.61 \%$. Development of fish sausage from Talang Queenfish performed by Yousefi and Moosavi-nasab (2014) also showed the ash content was within the range with $2.09 \%$.

\subsection{Cooking yield}

All the new formulated Keropok Lekors showed a weight loss in cooking yield \% ranged between 91.61$99.49 \%$. According to Santana et al. (2013), weight is gained in a product when the cooking yield is more than $100 \%$. While weight is loss when the cooking yield is less than $100 \%$. Nevertheless, the results were comparable to the study by Huda et al. (2012), where the cooking yield \% of commercial Malaysian fish sausage were recorded between 102.69-104.70\%. Another study conducted by Santana et al. (2013) reported that fish sausage formulated only with surimi powder and the addition of hydrocolloids were ranged between 95.59$99.14 \%$ of cooking yield.

In this study, KLCP showed a significant lower cooking yield $(91.61 \%)(\mathrm{p}<0.05)$ compared to KLT $(93.66 \%)$ and KLKL (99.33\%) (Table 3). Other new formulated Keropok Lekors resulted with higher cooking yield compared to KLT $(\mathrm{p}<0.05)$ but not significant against the KLKL $(p>0.05)$. KLKB showed the best quality of cooking yield \% compared to other formulated Keropok Lekors. The cooking yield in KLT was lower compared to other formulated Keropok Lekors due to the loss of water and fat in KLT. Since KLT have a higher amount of fish mince at $70 \%$ and low amount sago flour at $6.94 \%$ and tapioca starch at $6.94 \%$ compared to other formulations, the ability to retain water and fat after cooking is also low. Besides, when Keropok Lekor was cooked, the protein undergoes denaturation, which also results in the decrease in cooking yield percentage.

The substitution of legume flours into Keropok Lekors replacing tapioca starch did affect significantly the quality of cooking yield (\%). Keropok Lekor formulated with kidney bean flour had better cooking yield \% compared to Keropok Lekors formulated with tapioca starch. Furthermore, this study also proved that legume flours in Keropok Lekors have the ability to retain water and fat thus preventing from further cooking loss as compared to Keropok Lekors formulated with

Table 3. Cooking yield, texture properties and colour properties of Keropok Lekor with different types of flour.

\begin{tabular}{|c|c|c|c|c|c|c|c|c|}
\hline \multirow{2}{*}{$\begin{array}{l}\text { Keropok Lekor } \\
\text { formulations }\end{array}$} & \multirow{2}{*}{$\begin{array}{l}\text { Cooking } \\
\text { yield }(\%)\end{array}$} & \multicolumn{4}{|c|}{ Texture properties } & \multicolumn{3}{|c|}{ Colour properties } \\
\hline & & $\begin{array}{l}\text { Hardness } \\
(\mathrm{kg})\end{array}$ & $\begin{array}{c}\text { Springiness } \\
(\mathrm{mm})\end{array}$ & Cohesiveness & $\begin{array}{l}\text { Chewiness } \\
(\mathrm{kg} \mathrm{mm})\end{array}$ & $\mathrm{L}^{*}$ & $a^{*}$ & $\mathrm{~b}^{*}$ \\
\hline $\begin{array}{l}\text { Keropok Lekor } \\
\text { Terengganu (KLT) }\end{array}$ & $93.66 \pm 0.15^{b}$ & $5.84 \pm 0.54^{\mathrm{c}}$ & $0.49 \pm 0.052^{\mathrm{b}}$ & $0.23 \pm 0.006^{\mathrm{d}}$ & $0.66 \pm 0.12^{\mathrm{c}}$ & $\begin{array}{c}44.22 \pm \\
2.93^{\mathrm{a}}\end{array}$ & $\begin{array}{l}6.48 \pm \\
1.34^{\mathrm{ab}}\end{array}$ & $\begin{array}{c}23.36 \pm \\
0.90^{\mathrm{b}}\end{array}$ \\
\hline $\begin{array}{l}\text { Keropok Lekor } \\
\text { Kuala Lumpur } \\
\text { (KLKL) }\end{array}$ & $99.33 \pm 0.13^{\mathrm{a}}$ & $9.77 \pm 1.93^{b c}$ & $0.66 \pm 0.030^{\mathrm{a}}$ & $0.39 \pm 0.01^{\mathrm{a}}$ & $2.57 \pm 0.49^{\mathrm{b}}$ & $\begin{array}{l}40.98 \pm \\
1.35^{\mathrm{ab}}\end{array}$ & $\begin{array}{l}5.81 \pm \\
0.48^{\mathrm{ab}}\end{array}$ & $\begin{array}{r}17.60 \pm \\
0.92^{\mathrm{c}}\end{array}$ \\
\hline $\begin{array}{l}\text { Keropok Lekor } \\
\text { Chickpea (KLCP) }\end{array}$ & $91.61 \pm 1.05^{\mathrm{c}}$ & $6.84 \pm 1.28^{\mathrm{c}}$ & $0.45 \pm 0.037^{\mathrm{b}}$ & $0.27 \pm 0.008^{\mathrm{c}}$ & $0.84 \pm 0.08^{\mathrm{c}}$ & $\begin{array}{c}44.44 \pm \\
2.71^{\mathrm{a}}\end{array}$ & $\begin{array}{l}8.10 \pm \\
1.19^{\mathrm{a}}\end{array}$ & $\begin{array}{l}23.06^{ \pm} \\
1.32^{\mathrm{b}}\end{array}$ \\
\hline $\begin{array}{l}\text { Keropok Lekor } \\
\text { Soybean (KLSB) }\end{array}$ & $98.27 \pm 0.63^{\mathrm{a}}$ & $9.65 \pm 0.90^{\mathrm{bc}}$ & $0.43 \pm 0.03^{\mathrm{b}}$ & $0.23 \pm 0.004^{\mathrm{d}}$ & $0.98 \pm 0.15^{\mathrm{bc}}$ & $\begin{array}{r}40.58 \pm \\
1.63^{\mathrm{ab}}\end{array}$ & $\begin{array}{l}7.85 \pm \\
0.86^{\mathrm{a}}\end{array}$ & $\begin{array}{c}22.03 \pm \\
0.27^{\mathrm{b}}\end{array}$ \\
\hline $\begin{array}{l}\text { Keropok Lekor } \\
\text { Mung bean (KLMB) }\end{array}$ & $99.20 \pm 0.11^{\mathrm{a}}$ & $19.81 \pm 3.11^{\mathrm{a}}$ & $0.70 \pm 0.06^{\mathrm{a}}$ & $0.35 \pm 0.01^{b}$ & $4.97 \pm 1.34^{\mathrm{a}}$ & $\begin{array}{l}35.88 \pm \\
1.18^{\mathrm{b}}\end{array}$ & $\begin{array}{l}5.22 \pm \\
0.42^{\mathrm{b}}\end{array}$ & $\begin{array}{r}16.51 \pm \\
0.22^{\mathrm{c}}\end{array}$ \\
\hline $\begin{array}{l}\text { Keropok Lekor } \\
\text { Kidney bean } \\
\text { (KLKB) }\end{array}$ & $99.49 \pm 0.07^{\mathrm{a}}$ & $12.53 \pm 0.54^{b}$ & $0.45 \pm 0.008^{\mathrm{b}}$ & $0.24 \pm 0.01^{\mathrm{cd}}$ & $1.40 \pm 0.11^{\mathrm{bc}}$ & $\begin{array}{c}38.40 \pm \\
0.89^{\mathrm{b}}\end{array}$ & $\begin{array}{l}4.94 \pm \\
0.31^{\mathrm{b}}\end{array}$ & $\begin{array}{r}39.84 \pm \\
1.10^{\mathrm{a}}\end{array}$ \\
\hline
\end{tabular}

\footnotetext{
${ }^{\mathrm{a}-\mathrm{d}}$ Mean values with different letters in the same column are significantly different $(P<0.05) . \mathrm{L}^{*}$, lightness; $\mathrm{a}^{*}$, redness; $\mathrm{b}^{*}$,
} yellowness 
tapioca starch only.

\subsection{Colour analysis}

Table 3 shows that there were no significant differences ( $p>0.05)$ of $L^{*}$ (lightness) values between all Keropok Lekor formulations. However, KLMB has an $\mathrm{L}^{*}$ value of 35.88 , which was the darkest compared to other Keropok Lekor formulations. Previous literatures reported that the $\mathrm{L}^{*}$ values for fish sausage products were brighter in salmon sausages incorporated with salmon oil with values 69.02 (uncooked) and 64.71 (smoked) (Oliveira et al., 2013), fish sausage with surimi powder with values of 71.80-72.18 (Santana et al., 2013) and Malaysian commercial fish sausages with values within 58.73-79.56 (Huda et al., 2012). The new formulated Keropok Lekors were darker in colour (35.88 -44.44) as indicative of the $L^{*}$ value compared to normal fish sausages. This is due to the type of fish used during the processing of Keropok Lekor involving Longtail Tuna and Indian Mackerel. According to the Department of Primary Industries, New South Wales (2010), longtail tuna have a very dark flesh in colour. The dark flesh colour in longtail tuna meat is due to the presence of myoglobin meat pigment. Myoglobin is unstable compounds which can be oxidized when exposed to oxygen converting to brown metmyoglobin (Dolatowski and Olszak, 2007).

The $\mathrm{a}^{*}$ values showed no significant difference ( $p>0.05)$ between all the Keropok Lekor formulations ranging within 4.94-8.10. The $a^{*}$ values obtained from all Keropok Lekor formulations are comparable to the result shown by Huda et al. (2012) where Malaysian commercial fish sausage produced using tuna meat was 5.99. The $b^{*}$ values for all the Keropok Lekor formulations were in the bigger range between 16.5139.84 when compared to the findings by Al-Bulushi et al. (2013) ranging between 11.90-16.60. A study by Muthia et al. (2010) showed that duck sausages incorporated using different types of flours (tapioca, wheat, sago and potato) resulted with $b^{*}$ values ranged between 19.10-19.9. All the Keropok Lekor formulations showed a significantly higher $b^{*}$ values $(p<0.05)$ compared to KLKL (17.60) and KLMB (16.51) resulting in a more yellowish colour.

CIEL*a*b* colour analysis for Keropok Lekor formulated with legume flours proved no significant difference compared to Keropok Lekor formulated with tapioca starch. This finding showed that legume flours have the potential to replace tapioca starch in Keropok Lekors without altering the originality colour of Keropok Lekors.

\subsection{Texture profile analysis (TPA)}

Texture profile analysis is shown in Table 3, which involves hardness, springiness, cohesiveness, and chewiness for all Keropok Lekor formulations. KLMB $(19.81 \mathrm{~kg})$ was significantly harder $(\mathrm{p}<0.05)$ in texture compared to other formulated Keropok Lekors. Meanwhile, KLSB $(9.65 \mathrm{~kg})$ and KLCP $(6.84 \mathrm{~kg})$ showed no significant hardness compared to KLT and KLKL ( $>>0.05)$. Result for KLKB (12.53 kg) was significantly harder against KLT $(p<0.05)$ but not significant against KLKL $(p>0.05)$. Hardness is an important parameter when evaluating the textural properties of the sausage product. According to Muthia et al. (2010), textural properties of a sausage product are also affected by the type of different flours used due to the amylose and amylopectin structure and content of each flour and their granule size. Muthia et al. (2010) also added that flour with low swelling power will trap less water in starch molecule forming a harder texture. This study shows that KLMB and KLKB were harder in texture due to the low swelling power in the mung bean and kidney bean flours itself. Nevertheless, other Keropok Lekor formulated with legume flour found in KLCP and KLSB showed less hard in their textural properties when compared to KLKL. Therefore, this study indicates that the hardness of Keropok Lekor is affected by the different types of flours used and can be used as an indication in the quality of textural properties.

KLMB showed no significant difference $(\mathrm{p}>0.05)$ in springiness compared to KLKL, but both KLMB and KLKL showed higher springiness values $(\mathrm{P}<0.05)$ compared to KLT and other new formulated Keropok Lekors. According to Santana et al. (2013), springiness is affected by the quality of the protein. Therefore, the type of flour used in Keropok Lekor formulation has an effect on the springiness properties.

KLKL (0.39) has the highest cohesiveness value $(p<0.05)$ compared to KLT $(0.23)$ and other new formulated Keropok Lekors. KLMB (0.35) and KLCP $(0.27)$ were more cohesive $(\mathrm{p}<0.05)$ compared to KLT, while KLSB (0.23) and KLKB (0.24) showed no significant different $(p>0.05)$ compared to KLT. The range of the new formulated Keropok Lekor cohesiveness was between 0.23-0.35. Similar findings were obtained from Malaysian fish sausages with cohesiveness ranging between 0.28-0.42 (Huda et al., 2012). According to Farouk et al. (2002), the cohesiveness of fish products is affected by both sarcoplasmic and myofibrillar proteins during the mixing process. KLMB showed a significantly higher in chewiness $(p<0.05)$ compared to KLT and KLKL. While other new formulated Keropok Lekors chewiness were not significantly different $(\mathrm{p}>0.05)$ compared to KLT and KLKL except for KLCP where its value was lower 
Table 4. Sensory evaluations of Keropok Lekor with different types of flour.

\begin{tabular}{ccccccc}
\hline Attributes & $\begin{array}{c}\text { Keropok Lekor } \\
\text { Terengganu } \\
(\text { KLT })\end{array}$ & $\begin{array}{c}\text { Keropok Lekor } \\
\text { Kuala Lumpur } \\
(\text { KLKL) }\end{array}$ & $\begin{array}{c}\text { Keropok Lekor } \\
\text { Chickpea } \\
(\text { KLCP) }\end{array}$ & $\begin{array}{c}\text { Keropok Lekor } \\
\text { Soybean } \\
(\text { KLSB) }\end{array}$ & $\begin{array}{c}\text { Keropok Lekor } \\
\text { Mung bean } \\
(\text { KLMB) }\end{array}$ & $\begin{array}{c}\text { Keropok Lekor } \\
\text { Kidney bean } \\
(\text { KLKB) }\end{array}$ \\
\hline Colour & $4.50 \pm 1.43^{\mathrm{ab}}$ & $4.97 \pm 1.07^{\mathrm{a}}$ & $4.60 \pm 1.48^{\mathrm{ab}}$ & $4.47 \pm 1.36^{\mathrm{ab}}$ & $4.80 \pm 1.40^{\mathrm{a}}$ & $3.63 \pm 1.85^{\mathrm{b}}$ \\
Aroma & $4.83 \pm 1.09^{\mathrm{a}}$ & $4.93 \pm 1.17^{\mathrm{a}}$ & $4.93 \pm 1.17^{\mathrm{a}}$ & $4.60 \pm 1.28^{\mathrm{a}}$ & $4.83 \pm 1.32^{\mathrm{a}}$ & $5.07 \pm 1.02^{\mathrm{a}}$ \\
Chewiness & $4.63 \pm 1.27^{\mathrm{ab}}$ & $5.20 \pm 1.19^{\mathrm{a}}$ & $4.60 \pm 1.30^{\mathrm{ab}}$ & $4.00 \pm 1.44^{\mathrm{b}}$ & $4.77 \pm 1.46^{\mathrm{ab}}$ & $4.43 \pm 1.33^{\mathrm{ab}}$ \\
Juiciness & $5.07 \pm 1.11^{\mathrm{a}}$ & $5.07 \pm 1.08^{\mathrm{a}}$ & $4.20 \pm 1.30^{\mathrm{ab}}$ & $3.83 \pm 1.23^{\mathrm{b}}$ & $4.67 \pm 1.32^{\mathrm{ab}}$ & $4.20 \pm 1.13^{\mathrm{ab}}$ \\
Aftertaste & $4.63 \pm 1.19^{\mathrm{ab}}$ & $5.03 \pm 1.25^{\mathrm{a}}$ & $4.00 \pm 1.31^{\mathrm{b}}$ & $3.77 \pm 1.22^{\mathrm{b}}$ & $4.50 \pm 1.38^{\mathrm{ab}}$ & $4.10 \pm 1.27^{\mathrm{ab}}$ \\
Overall & $4.47 \pm 1.28^{\mathrm{abc}}$ & $5.23 \pm 1.19^{\mathrm{a}}$ & $4.33 \pm 1.32^{\mathrm{abc}}$ & $3.63 \pm 1.38^{\mathrm{c}}$ & $4.70 \pm 1.26^{\mathrm{ab}}$ & $4.03 \pm 1.35^{\mathrm{bc}}$ \\
acceptability & & & & & & \\
\hline
\end{tabular}

${ }^{\mathrm{a}-\mathrm{c}}$ Mean values with different letters in the same row are significantly different $(P<0.05)$.

than KLKL $(\mathrm{p}<0.05)$. Santana et al. (2013) reported that chewiness of a fish sausage product might be affected by the protein content. This study proves that legume flours substituted into Keropok Lekors did change the textural properties in terms of hardness, springiness and cohesiveness when compared to Keropok Lekor formulated with tapioca starch.

\subsection{Sensory evaluation}

The sensory evaluation conducted involves acceptance test, which has five attributes (colour, aroma, chewiness, juiciness, and aftertaste) and overall acceptability as shown in Table 4. There was no significant different $(p>0.05)$ of the scores given for aroma between all the Keropok Lekors. All the Keropok Lekors resulted with no significant colour preference $(\mathrm{p}<0.05)$ against the controls except for KLKB. A previous study conducted by Serdaroğlu et al. (2005) indicated that meatballs incorporated with different flours (black-eyed pea, chickpea, lentil and rusk) did not have any significant difference on the colour and flavour. This proves that the aroma and colour preference of Keropok Lekor formulated with legume flours were acceptable and have similar value scores given by panelists when compared to Keropok Lekor formulated with tapioca starch.

The chewiness and juiciness also showed no significant difference $(p>0.05)$ except for KLSB. The chewiness attribute was given higher scores by panelists for KLKL (5.20) and KLMB (4.77) compared to other Keropok Lekor formulations. Relatively, analysis using Texture Profile Analysis provided higher chewiness in KLKL (2.57 kg mm) and KLMB (4.97 kg mm) compared to other formulations. The juiciness attribute was given higher scores for KLT (5.07), KLKL (5.07) and KLMB (4.67) compared to other Keropok Lekor formulations. According to Santana et al. (2013), the soft texture of surimi powder fish sausage leads to the release of fluid after chewing compared to fish sausage formulated with hydrocolloids. This is agreeable for the higher score of chewiness in KLT since the moisture content $(62.42 \%)$ is higher compared to other Keropok
Lekor formulations. This study shows that the legume flours were able to improve the chewiness and juiciness of Keropok Lekors without altering the sensory attributes.

While, the aftertaste attributes were similar $(\mathrm{p}>0.0)$ between KLMB, KLKB and the controls. The panellists preferred KLMB and KLCP for overall acceptability among the new formulated Keropok Lekors produced, where they did not differ significantly $(\mathrm{p}>0.05)$ against the positive control (KLT) and the negative control (KLKL). Therefore, Keropok Lekors formulated with legume flours did not have an effect on overall acceptability of Keropok Lekor compared to Keropok Lekor formulated with tapioca starch since almost similar scores were given. This proves that legume flours have the potential in replacing tapioca starch in Keropok Lekors and are accepted by consumers.

\section{Conclusion}

All the legume flours used as the substitute for tapioca starch have shown potential to be used in producing Keropok Lekor. However, KLMB and KLCP had the nearest physicochemical properties compared to KLT (positive control) and was better in many aspects compared to KLKL (negative control). The acceptance of KLMB and KLCP in term of sensory, which were almost similar to KLKL provides a good indicator that they could be produced without consumers noticing the differences compared to the original KLKL. As an advantage, the nutrient content especially protein found in KLSB is higher compared to Keropok Lekor (KLT) produced in east coast states, which has a high amount of fish content. It can be concluded that this study was successful in finding an alternative way to enhance the nutritional content of Keropok Lekor by substituting tapioca starch with legume flours thus retaining its quality attributes.

\section{Conflict of Interest}

The authors declare no conflict of interest. 


\section{Acknowledgements}

The authors acknowledge partial financial support by the Master of Food Technology (MoFT) course at the Faculty of Food Science and Technology, UPM.

\section{References}

Abbas, M. and Shah, H.U. (2007). Proximate and mineral composition of mung bean. Sarhad Journal of Agriculture (Pakistan), 23(2), 2005-2008.

Ahmad, W.I.W., Rahman, A.A. and Ismail, Z. (2011). Informal sector in rural areas: Socio-demographic profile of traditional food industry entrepreneur in Kelantan and Terengganu, Malaysia. International Journal of Humanities and Social Science, 1(5), 221227.

Alajaji, S.A. and El-Adawy, T.A. (2006). Nutritional composition of chickpea (Cicer arietinum L.) as affected by microwave cooking and other traditional cooking methods. Journal of Food Composition and Analysis, 19(8), 806-812. https://doi.org/10.1016/ j.jfca.2006.03.015

Al-Bulushi, I.M., Kasapis, S., Dykes, G.A., Al-Waili, H., Guzaini, N. and Al-Oufi, H. (2013). Effect of frozen storage on the characteristics of a developed and commercial fish sausages. Journal of Food Science and Technology, 50(6), 1158-1164. https:// doi.org/10.1007/s13197-011-0441-x

AOAC. (2002). Official Methods of Analysis. $16^{\text {th }}$ ed. Washington: Association of Official Analytical Chemists.

Arab, E.A.A., Helmy, I.M.F. and Bareh, G.F. (2010). Nutritional Evaluation and Functional Properties of Chickpea (Cicer arietinum L.) Flour and the Improvement of Spaghetti. Journal of American Science, 6(10), 1055-1072.

Audu, S.S. and Aremu, M.O. (2011). Effect of Processing on Chemical Composition of Red Kidney Bean (Phaseolus vulgaris L.) Flour. Pakistan Journal of Nutrition, 10(11), 1069-1075. https:// doi.org/10.3923/pjn.2011.1069.1075

Blessing, I.A. and Gregory, I.O. (2010). Effect of processing on the proximate composition of the dehulled and undehulled mungbean [Vigna radiata (L.) Wilczek] flours. Pakistan Journal of Nutrition, 9 (10), 1006-1016. https://doi.org/10.3923/ pjn.2010.1006.1016

Chaudhary, R. and Sharma, S. (2013). Conventional nutrients and antioxidants in red kidney beans (Phaseolus vulgaris L.): An explorative and product development endeavour. Annals Food Science and Technology, 14(2), 275-285.

Department of Primary Industries, New South Wales.
(2010). Retrieved on March 20, 2015 from Department of Primary Industries, New South Wales website: www.dpi.nsw.gov.au/_data/assets/ pdf_file/0004/375907/Longtail-Tuna.pdf

Dolatowski, Z.J. and Olszak, M. (2007). Effect of k Carrageenan on Colour Stability of Model Products with Different Levels of Fat. Electronic Journal of Polish Agricultural Universities, 10(1), 1-9.

Embong, M.S., Ishak, S., Rahim, M.A.A. and Shafie, N. (1990). The effect of various treatments on the dehydration and fish sausage. In Seminar on Advances in Food Research, Serdang, Selangor (Malaysia), 19-20 Nov 1990. Canada: Impact Publishing.

Farouk, M.M., Wieliczko, K., Lim, R., Turnwald, S. and MacDonald, G.A. (2002). Cooked sausage batter cohesiveness as affected by sarcoplasmic proteins. Meat Science, 61(1), 85-90. https://doi.org/10.1016/ S0309-1740(01)00168-1

Hajeb, P., Jinap, S., Ismail, A., Fatimah, A.B., Jamilah, B. and Rahim, M.A. (2009). Assessment of mercury level in commonly consumed marine fishes in Malaysia. Food Control, 20(1), 79-84. https:// doi.org/10.1016/j.foodcont.2008.02.012

Hatta, W.N.N.W.M. (2015). The Authentic of "Keropok Lekor" Process. Arts and Design Study, 27, 1-7.

Hayat, I., Ahmad, A., Ahmed, A., Khalil, S. and Gulfraz, M. (2014). Exploring the Potential of Red Kidney Beans (Phaseolus vulgaris L.) to Develop Protein Based Product for Food Applications. The Journal of Animal and Plant Sciences, 24(3), 860-868.

Hayes, J.E., Desmond, E.M., Troy, D.J., Buckley, D.J. and Mehra, R. (2005). The effect of whey proteinenriched fractions on the physical and sensory properties of frankfurters. Meat Science, 71(2), 238243. https://doi.org/10.1016/j.meatsci.2005.03.005

Hegstad, H.G. (2008). Nutritional and Health benefits of Soybean. Soy Protein Quality Evaluation Report, Food and Agriculture Organization of the United Nations. Food and Nutrition Paper No. 71. Rome, Italy: FAO

Huda, N., Alistair, T.L.J., Lim, H.W. and Nopianti, R. (2012). Some quality characteristics of Malaysian commercial fish sausage. Pakistan Journal of Nutrition, 11(8), 700-705. https://doi.org/10.3923/ pjn.2012.798.803

Jamilah, B. (1983). Keropok Lekor - Boiling and Steaming Methods of Processing. Pertanika, 6(3), 56 -60 .

Kenawi, M.A., Abdelsalam, R.R. and El-Sherif, S.A. (2009). The effect of mung bean powder, and/or low -fat soy flour as meat extender on the chemical, 
physical, and sensory quality of buffalo meat product. Biotechnology in Animal Husbandry, 25(56), 327-337. https://doi.org/10.2298/BAH0906327K

Lakshmisha, I.P., Ravishankar, C.N., Ninan, G., Mohan, C.O. and Gopal, T.K.S. (2008). Effect of Freezing Time on the Quality of Indian Mackerel (Rastrelliger kanagurta). Sensory and Food Quality, 73(7), 345353.

Malaysian Fisheries Department. (2014). Retrieved on May 16, 2015 from Department of Fisheries Website: http://www.dof.gov.my/index.php/pages/ view/143.

Murad, N.S., Ismail, M.A., Nor-Khaizura, M.A.R. and Ismail-Fitry, M.R. (2017). The Effect of Mixing Time and Mixing Sequence during Processing on the Physicochemical and Sensory Properties of Keropok Lekor. Journal of Science and Technology, 9(4), 8895.

Muthia, D., Nurul, H., and Noryati, I. (2010). The effects of tapioca, wheat, sago and potato flours on the physicochemical and sensory properties of duck sausage. International Food Research Journal, 17 (4), 877-884.

Odiase, O.M., Igene, J.O., Evivie, S.E. and Ebabhamiegbebho, P.A. (2013). Determination and sensory evaluation of soy flour-meat combinations in the production of meatballs. Journal of Applied and Natural Science, 5(2), 482-487. https:// doi.org/10.31018/jans.v5i2.357

Oliveira, A.C.M., Himelbloom, B.H., Montazeri, N., Davenport, M., Biceroglu, H., Brenner, K.A., Thomas, S.R. and Crapo, C.A. (2014). Development and characterization of fish sausages supplemented with salmon oil. Journal of Food Processing and Preservation, 38(4), 1641-1652. https:// doi.org/10.1111/jfpp.12126

Paul, T., Mozumder, N.H.M.R., Sayed, M.A. and Akhtaruzzaman, M. (2011). Proximate Compositions, Mineral Contents and Determination of Protease Activity from Green Gram. Bangladesh Research Publications Journal, 5(3), 207-213.

Perera, M.P.M.S.H., Sivakanesan, R., Abeysekara, D.T.D.J. and Sarananda, K.H. (2013). Sensory Evaluation and Available Carbohydrate Content of Soy Flour Incorporated Cereal Based Traditional Sri Lankan Breakfast Foods. International Journal of Research in Agriculture and Food Sciences, 1(4), 2013-2014.

Prabpree, R. and Pongsawatmanit, R. (2011). Effect of tapioca starch concentration on quality and freezethaw stability of fish sausage. Kasetsart Journal Natural Science, 45, 314-324.
Rahman, M.S., Al-Waili, H., Guizani, N. and Kasapis, S. (2007). Instrumental-sensory evaluation of texture for fish sausage and its storage stability. Fisheries Science, 73(5), 1166-1176. https://doi.org/10.1111/ j.1444-2906.2007.01449.x

Santana, P., Huda, N. and Yang, T.A. (2013). The Addition of Hydrocolloids (Carboxymethylcellulose, Alginate and Konjac) to Improve the Physicochemical Properties and Sensory Characteristics of Fish Sausage Formulated with Surimi Powder. Turkish Journal of Fisheries and Aquatic Sciences, 13 (4), 561-569. https:// doi.org/10.4194/1303-2712-v13_4_01

Seechamnanturakit, V. and Karrila, T.T. (2015). Physicochemical properties of blends from tapioca starch and waxy rice for use as functional food. European International Journal of Science and Technology, 4(9), 26-36.

Serdaroğlu, M., Yıldız-Turp, G. and Abrodímov, K. (2005). Quality of low-fat meatballs containing Legume flours as extenders. Meat Science, 70(1), 99 -105. https://doi.org/10.1016/j.meatsci.2004.12.015

Verma, M.M., Ledward, D.A. and Lawrie, R.A. (1984). Utilization of chickpea flour in sausages. Meat Science, 11(2), 109-121. https:// doi.org/10.1016/0309-1740(84)90009-3

Wani, S.A. and Kumar, P. (2014). Comparative Study of Chickpea and Green Pea Flour Based on Chemical Composition, Functional and Pasting Properties. Journal of Research and Technology, 2(3), 124-129.

Tang, J.Y.H., Mohd-Noor, N.H., Mazlan, N., Chieng, C., Abu-bakar, C.A. and Son, R. (2014). Survival of Vibrio cholerae $\mathrm{O} 1$ and Vibrio parahaemolyticus in fried and boiled Malaysian fish sausage. Food Control, 41, 102-105. https://doi.org/10.1016/ j.foodcont.2014.01.004

Yousefi, A. and Moosavi-Nasab, M. (2014). Textural and chemical attributes of sausages developed from Talang Queenfish (Scomberoides commersonnianuus) mince and surimi. Iranian Journal of Fisheries Sciences, 13(1), 228-241. 